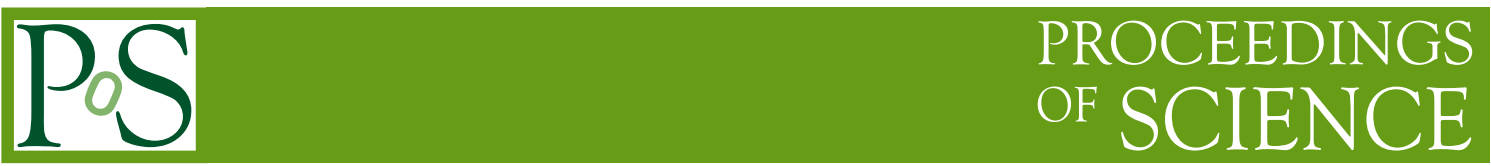

\title{
Recent solar and geo- $v$ results from Borexino
}

\section{S. Zavatarelli*}

I.N.F.N - Sezione di Genova, Vie Dodecanneso 33, 16146 Genova (Italy)

E-mail: zavatare@ge.infn.it

M. Agostini, K. Altenmüller, S. Appel, V. Atroshchenko, G. Bellini, J. Benziger, D. Bick, G. Bonfini, D. Bravo, B. Caccianiga, F. Calaprice, A. Caminata, M. Carlini, P. Cavalcante, A. Chepurnov, K. Choi, S. Davini, A. Derbin, L. Di Noto, I. Drachnev, A. Etenko, K. Fomenko, D. Franco, F. Gabriele, C. Galbiati, C. Ghiano, M. Giammarchi, M. Göger-Neff, A. Goretti, M. Gromov, C. Hagner, E. Hungerford, Aldo lanni, Andrea lanni, A. Jany, K. Jedrzejczak, D. Jeschke, V. Kobychev, D. Korablev, G. Korga, D. Kryn, M. Laubenstein, B. Lehnert, E. Litvinovich, F. Lombardi, P. Lombardi, L. Ludhova, G. Lukyanchenko, I. Machulin, S. Manecki, W. Maneschg, G. Manuzio, S. Marcocci, E. Meroni, M. Meyer, L. Miramonti, M. Misiaszek, M. Montuschi, P. Mosteiro, V. Muratova, B. Neumair, L. Oberauer, M. Obolensky, F. Ortica, M.

Pallavicini, L. Papp, A. Pocar, G. Ranucci, A. Razeto, A. Re, A. Romani, R. Roncin, N. Rossi, S. Schrönert, D. Semenov, M. Skorokhvatov, O. Smirnov, A. Sotnikov, S. Sukhotin, Y. Suvorov, R. Tartaglia, G. Testera, J. Thurn, M. Toropova, E. Unzhakov, A. Vishneva, R.B. Vogelaar, F. von Feilitzsch, H. Wang, S. Weinz, J. Winter, M. Wojcik, M. Wurm, Z. Yokley, O. Zaimidoroga, S. Zavatarelli, K. Zuber, and G. Zuzel (Borexino Collaboration)

\footnotetext{
Neutrinos are impervious to the strong and electromagnetic forces and their minuscule weak interactions allow them to freely escape from the deep of the stars or of the Earth bringing precious information about regions otherwise inaccessible.

Borexino is a large, unsegmented liquid-scintillator calorimeter in operation at the Laboratori Nazionali del Gran Sasso (LNGS) in Italy, since 2007. The record-high radio-purity is the basis of the outstanding achievements obtained by the experiment over the 10-year long data taking. This contribution is aimed at reviewing the most recent Borexino results in the field of the solar and geo-neutrino physics, namely the measure of the fundamental pp solar neutrino flux, which is the direct probe of solar thermonuclear fusion reactions, and the measure of the geo-neutrino signal with its geophysical implications.
}

Neutrino Oscillation Workshop

4 - 11 September, 2016

Otranto (Lecce, Italy)

${ }^{*}$ Speaker. 


\section{Neutrino physics and Borexino}

Neutrinos and their matter counterpart- antineutrinos - are helping scientist to accomplish a manifold range of applications beyond fundamental physics: in particular they are unic tools to precisely constrain the energy production inside the Sun or to establish the amount of $U$ and $T h$ in the Earth, thus settling a long discussion about Earth's internal heat sources initiated almost 200 years ago.

The Sun is an intense source of neutrinos, produced in nuclear reactions of the p-p chain and of the CNO cycle [1]. Measurements of the individual neutrino fluxes are of paramount importance for both particle physics and astrophysics. In particular, because of the strict relation between the photon and neutrino luminosity, the measurement of the total neutrino luminosity provides a test of the stability of the Sun over a scale of $10^{5}$ years [1], the time needed for the radiation born at the center of the Sun to arrive to its surface. In the case of an agreement between the two measurements, also other unknown sources of the solar energy may be constrained.

The main neutrino sources in the Sun are the pp and ${ }^{7} \mathrm{Be}$ reactions[1], providing roughly $91 \%$ and $7 \%$ of the total flux respectively[1]. Borexino measured in 2011 the ${ }^{7} \mathrm{Be}$ neutrino flux with 5\% precision [2], but a direct measurement the pp-neutrino flux was still missing up to the most recent years, and only indirect estimations based on joined analysis of all the solar experiment results were available. In 2014 the Borexino collaboration pursued an effort to extract the pp-neutrino flux from a careful analysis of the event spectrum in the lowest energy range, namely between 165-590 $\mathrm{keV}$. The phase II data, registered after the detector purification campaigns in 2010, were used for this analysis for a total of 408 live days from January 2012 to May 2013 [3] .

The pp neutrino energy spectrum extends up to $420 \mathrm{keV}$, yielding in the scattering process a maximum electron recoil energy of $264 \mathrm{keV}$ : particularly relevant to this analysis are the backgrounds due to ${ }^{14} \mathrm{C}$, an $\beta$ emitter intrinsic to the organic liquid scintillator whose energy spectrum extends up to $156 \mathrm{keV}$ and its pile-up. ${ }^{14} \mathrm{C}$ and pp neutrinos exhibit however different energy spectra (see Fig.1-left) and independent analysis methods were developed to constrain the ${ }^{14} \mathrm{C}$ background. The signal of the pp solar neutrinos was extracted from the data through a fit of the energy spectrum of the events collected in the $165-590 \mathrm{keV}$ energy window: the collected statistics in the low energy bins was very high, demanding an accurate modeling for the detector response, verified with Monte Carlo simulations, in order to limit the systematical uncertainty to a value comparable to the statistical fluctuations of $0.2 \%[3]$. The development of such a precise model was one of the main goals of the analysis. The solar pp-neutrino flux measured by Borexino[3] was of $\Phi_{p p}($ Borex $)$ $=(6.6 \pm 0.7) 10^{10} \mathrm{~cm}^{-2} \mathrm{~s}^{-1}$, in a good agreement with the expectation from solar models, $6.0(1.000$ $\pm 0.006) 10^{10} \mathrm{~cm}^{-2} \mathrm{~s}^{-1}[1]$ and also with the combined value of the radiochemical and other solar experiments $\Phi_{p p}($ other $)=(6.14 \pm 0.61) 10^{10} \mathrm{~cm}^{-2} \mathrm{~s}^{-1}$ [4]. The total energy production in the solar reactions calculated from the corresponding neutrino fluxes is $(4.04 \pm 0.28) 10^{26} \mathrm{~W} \mathrm{~s}^{-1}$ coherent with a total photon luminosity: $\mathrm{L}_{\odot}=3.84610^{26} \mathrm{~W} \mathrm{~s}^{-1}$ [5]. If one assumes that other unknown sources do exist, their total power cannot exceed $0.1510^{26} \mathrm{~W} \mathrm{~s}^{-1}$ with $90 \%$ probability . We can conclude that Borexino provided an independent measurement of the Solar pp- neutrino flux and demonstrated that no more than $4 \%$ of the total energy production is left for the unknown energy sources, confirming the thermonuclear energy production in the Sun. 


\section{Geo-neutrinos: unlocking the deep Earth's secrets}

The Earth emits a tiny heat flux with an average value of $\sim 75 \mathrm{~mW} \mathrm{~m}^{-2}$, much smaller than the radiation coming from the Sun $\left(1.4 \mathrm{~kW} \mathrm{~m}^{-2}\right)$. When integrated over the Earth's surface, anyway this tiny flux translates into a huge heat flow, $\mathrm{H}=47 \pm 2 \mathrm{TW}$ (stat) [6], the equivalent of ten thousand nuclear power plants. Geophysicists have created models that predict that $\sim 70 \%$ of the total surface heat flux is from the decay of radioactive nuclides, while geochemists think $\sim 25 \%$. The spread in the predictions is really large and the overall picture uncertain: other possible heat sources are, as example, tidal frictions, thermal contraction or the gravitational energy released after the earthquakes of by the primordial core formation.

Geo-neutrinos are the electron antineutrinos released in the decays of radioactive elements distributed through the Earth and known as Heat Producing Elements (HPE): they are mainly produced in the $\beta$ decay of ${ }^{40} \mathrm{~K}$ and of several nuclides in the chains of ${ }^{238} \mathrm{U}$ and ${ }^{232} \mathrm{Th}$. The emitted heat is in a well fixed ratio with the total mass of HPE inside the Earth, thus the observation of geoneutrinos is "the method" to directly measure the HPE abundances into the deep Earth. Currently, only two large-volume, liquid-scintillator neutrino experiments, KamLAND in Japan [7, 8, 9] and Borexino in Italy $[10,11,12]$, have been able to measure the geo-neutrino signal. Antineutrinos are detected in liquid organic scintillators by means of the Inverse Beta Decay (IBD) reaction on protons: $\bar{v}_{e}+p \rightarrow e^{+}+n$, a process having a threshold of $1.8 \mathrm{MeV}$. Only geo-neutrinos from $\mathrm{U}$ an Th decay chains are enough energetic to be detected. Borexino made the first observation of the geo-neutrino signal already in 2010 with a statistical significance of more than $4 \sigma$ [10], and it has recently released an updated result [12] based on 2,056 days of data taking in the period December 2007 to March 2015 and corresponding to a total efficiency-corrected exposure of $907 \pm 44$ ton/yr. In the IBD reaction the coincidence between the prompt positron signal and the delayed 2.2 $\mathrm{MeV}$ gamma due to neutron capture on protons makes the interaction signature very clean. Energy, space/time correlation and pulse shape cuts are applied for an overall detection efficiency of $(84.2$ $\pm 1.5) \%: 77 \bar{v}_{e}$ candidates passing all the selection cuts have been identified [12]. The estimated background, that mimics $\bar{v}_{e}$ candidates, amounts to only $0.78_{-0.10}^{+0.13}$ events.

Antineutrinos from nuclear reactor power plants are indeed the main background to the geoneutrino measurements. They are more energetic with respect to the geo-neutrino and therefore they can be experimentally disentangled thanks to a fit of prompt event energies: in Fig.1-right the selected candidates and the fit results are shown. Using the value ratio for the masses of Th and $\mathrm{U}, \mathrm{m}(\mathrm{Th}) / \mathrm{m}(\mathrm{U})=3.9$, suggested by the chondritic model, the best fit yields $\mathrm{S}_{\text {geo }}=23.7$ \pm 65.57 (stat) \pm 0.96 (sys) events. The measured geo-neutrino signal corresponds to fluxes at the detector from decays in the $U$ and Th chains of $\Phi(\mathrm{U})=(2.7 \pm 0.7) 10^{6} \mathrm{~cm}^{-2} \mathrm{~s}^{-1}$ and $\Phi(\mathrm{Th})=(2.3 \pm$ 0.6) $10^{6} \mathrm{~cm}^{-2} \mathrm{~s}^{-1}$, respectively. Borexino observes geo-neutrinos with $5.9 \sigma$ significance (i.e., well above the "discovery" limit) and rejects the null hypothesis with a probability equal to $3.610^{-9}$. According to geological studies among the 77 candidates, $12.8 \pm 7.0$ events are expected to have been induced by the crust radioactivity: the hypothesis of $\mathrm{S}_{\text {mantle }}=0$ is rejected by the present result at $98 \%$ C.L. It is possible to extract from the measured geo-neutrino signal the Earth's radiogenic heat power, which is essential for understanding the plate tectonics and mantle convection. The uncertain distribution of HPE's in the deep Earth has to be included: by following a very conservative approach one can demonstrate that the present result corresponds to 23-36 TW of radiogenic 
heat [12] According to the Borexino best fit result the radiogenic heat production supplies $\sim 70 \%$ of the emitted surface flux but the large uncertainty is still preventing the selection of precise Earth models. In conclusion geo-neutrinos may be the key tool to support new discoveries about the deep Earth.
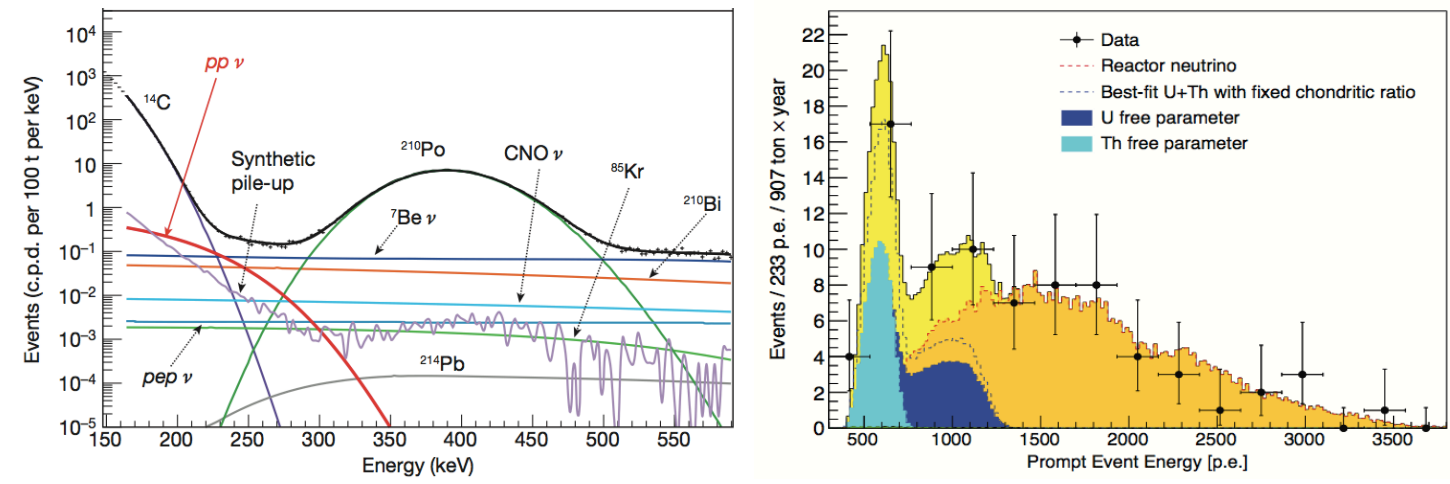

Figure 1: Left: Borexino energy spectrum between 165 and $590 \mathrm{keV}$ : the best fit $p p$ neutrino component is shown in red (see [3]). Right: prompt light yield spectrum, in units of photoelectrons (p.e.), of antineutrino candidates and best-fit. The best-fit shows the total contribution of geoneutrino, reactor neutrino and background (yellow colored area) and reactor neutrino (orange colored area) assuming the chondritic ratio. The result of a separate fit with $\mathrm{U}$ (blue colored area) and Th (light-blue colored area) set as free and independent parameters is also shown[12].

\section{References}

[1] A. Serenelli, W.C. Haxton, C. Pena-Garay, Astrophys. J. 743 (2011) 1.

[2] G. Bellini et al. (Borexino collaboration), Phys. Rev. Lett. 107 (2011) 141302.

[3] G. Bellini et al. (Borexino collaboration), Nature 512 (2014) 383-386.

[4] A. Ianni, Physics of the Dark Universe 4 (2014) 44.

[5] C. Fröhlich and J. Lean, Geophysical Research Letters 25 (1998) 4377.

[6] J. H. Davies and D. R. Davies, Solid Earth 1 (2010) 5.

[7] T. Araki et al. (KamLAND collaboration), Nature 436 (2005) 499.

[8] S. Abe et al. (KamLAND collaboration), Phys. Rev. Lett. 100 (2008) 221803.

[9] A. Gando et al. (KamLAND collaboration), Phys. Rev. D 88 (2013) 033001.

[10] G. Bellini et al. (Borexino collaboration), Phys. Lett. B 687 (2010) 299.

[11] G. Bellini et al. (Borexino collaboration), Phys. Lett. B 722 (2013) 295.

[12] M. Agostini et al. (Borexino collaboration), Phys. Rev. D 92 (2015) 031101(R). 